\title{
Disease progression patterns of bevacizumab responders with recurrent malignant gliomas
}

\author{
JU-HWI KIM ${ }^{1}$, TAE-YOUNG JUNG ${ }^{1}$, EU CHANG HWANG ${ }^{2}$, SUNG-HOON JUNG $^{3}$, SHIN JUNG ${ }^{1}$, \\ IN-YOUNG KIM ${ }^{1}$, WOO-YOUL JANG ${ }^{1}$, KYUNG-SUB MOON ${ }^{1}$, KYUNG-HWA LEE $^{4}$ and SEUL-KEE KIM ${ }^{5}$ \\ Departments of ${ }^{1}$ Neurosurgery, ${ }^{2}$ Urology, ${ }^{3}$ Hemato-oncology, ${ }^{4}$ Pathology and ${ }^{5}$ Radiology, Chonnam \\ National University Research Institute of Medical Sciences, Chonnam National University Hwasun \\ Hospital and Medical School, Gwangju, Chonnam 519-763, Republic of Korea
}

Received May 10, 2016; Accepted March 3, 2017

DOI: $10.3892 / \mathrm{ol} .2017 .6630$

\begin{abstract}
Tumor progression in patients with recurrent malignant glioma who respond to bevacizumab (BEV) is difficult to assess. The current study reviewed the clinical and radiological results of patients following a BEV-based chemotherapy regimen, and evaluated disease progression patterns in patients who responded to BEV therapy. From August 2011 to November 2015, 24 patients (18 glioblastoma cases and 6 anaplastic astrocytoma cases) were treated with BEV-based chemotherapy. In total, 6 patients were treated with BEV alone and 18 patients were treated with BEV combined with irinotecan. The male-female ratio was 10:14, and the median age was 47.5 years (range, 29-69). Patient performance status (PS) was classified using the Eastern Cooperative Oncology Group PS scores as follows: PS $1(n=3)$, PS 2 (n=9), PS 3 $(n=9)$ and PS $4(n=3)$. Treatment-associated complications were also analyzed according to the National Cancer Institute Common Terminology Criteria for Adverse Events version 4.0. Treatment responses were estimated using the Response Assessment in Neuro-Oncology Criteria. Progression-free survival (PFS) following treatment, patterns of disease progression and overall survival following treatment failure were also analyzed. The median PFS was 2.8 months (range, 0.6-10.1). In total, 2 patients did not continue treatment due to rectal bleeding and severe hematologic toxicity. Amongst the BEV responders $(n=16,72.7 \%)$, there was clinical deterioration without significant radiological progression in 2 patients $(n=2$, $12.5 \%$ ). Radiological progression of non-enhancing lesions without enhancement flare-ups was observed in 6 patients
\end{abstract}

Correspondence to: $\mathrm{Dr}$ Tae-Young Jung, Department of Neurosurgery, Chonnam National University Research Institute of Medical Sciences, Chonnam National University Hwasun Hospital and Medical School, 322 Seoyang-ro, Gwangju, Chonnam 519-763, Republic of Korea

E-mail: jung-ty@chonnam.ac.kr

Key words: bevacizumab, clinical, radiological, progression, malignant glioma
$(42.9 \%)$. A total of 3 of those lesions were diffuse and 3 were focal. Increased lesion enhancement was observed in 8 patients (57.1\%). Of the non-responders $(n=6,27.3 \%)$, diffuse enlargement of non-enhancing lesions was detected in 2 patients and an increase in lesion enhancement occurred in 4 patients. $\mathrm{BEV}$ complete responders $(\mathrm{n}=3)$ radiologically progressed with enlarged T2/fluid attenuation inversion recovery lesions without enhancement, followed by enhancement flare-ups. Following BEV treatment failure, 8 patients received a number of adjuvant treatments and the overall survival was 4.5 months (range, 0.4-34.0). Clinical symptoms and radiological alterations of non-enhancing lesions must be evaluated in order to assess tumor progression in the BEV responders, particularly in patients who have achieved complete remission.

\section{Introduction}

Bevacizumab (BEV; Avastin ${ }^{\circledR}$ ) is a monoclonal humanized antibody that targets vascular endothelial growth factor (VEGF). BEV-based chemotherapy has resulted in an objective radiological response of 19.6-37.8\% and a median response duration of 3.0-11.0 months in high-grade gliomas (1-4). Even with the high radiological response rates to anti-angiogenic therapy, the majority of patients develop progressive disease within a year of treatment. Following treatment, alternative VEGF-independent pro-angiogenic growth factors may be activated, resulting in tumor resistance and aggressive tumor characteristics $(5,6)$.

Following anti-angiogenic treatment, the blood-brain barrier may be restored (5). Contrast enhancement in T1-weighted magnetic resonance images (MRI) and hyperintensity in T2/fluid attenuation inversion recovery (FLAIR) images may be reduced, which causes the early detection of tumor progression to be challenging. In order to evaluate responses to an anti-angiogenic treatment such as BEV, MRI results, including T1-weighted images with contrast enhancement and T2/FLAIR images, and clinical status must be considered according to the Response Assessment in Neuro-Oncology (RANO) criteria (7). However, there are certain vague aspects in determining tumor progression that only use the RANO criteria as this criteria does not assess the degree of T2/FLAIR alteration. 
Tumor progression is difficult to identify in BEV responders with recurrent malignant glioma. Certain patients exhibit clinical deterioration without definitive radiological progression, and other patients exhibit progressive enlargement of non-enhancing lesions without neurological deterioration. Therefore, the present study reviewed clinical and radiological results following BEV-based chemotherapy, and evaluated patterns of disease progression in the BEV responders.

\section{Materials and methods}

Patient clinical and tumor characteristics. Between August 2011 and November 2015, surgery was performed on 6 patients with anaplastic astrocytoma and 18 patients with glioblastoma, which were classified according to the World Health Organization criteria 2007 (8). The Institutional Review Board of Chonnam National University Hwasun Hospital (CNUHH-2016-052; Chonnam, Korea) approved the current study. Patient characteristics are summarized in Table I. The male-female ratio was 10:14 and the median age was 47.5 years (range, 29-69).

As an initial study, the extent of tumor removal was assessed using postoperative MRI, and removal using three subgroups was classified as follows: Gross total resection (no obvious residual tumor), subtotal resection (residual tumor, $<50 \%$ ) and partial resection (residual tumor, $\geq 50 \%$ ). Tumors were completely removed from 6 patients $(25.0 \%)$ and partially removed from 13 patients $(54.2 \%)$. Biopsies were performed in 5 patients $(20.8 \%)$. A total of 19 patients underwent surgery followed by radiotherapy and concomitant temozolomide chemotherapy. In total, 5 patients received surgery followed by radiotherapy alone. The median radiation dose was $59.4 \mathrm{~Gy}$ (range, 50.4-60.0). As adjuvant treatment prior to BEV-based chemotherapy, surgical resection was performed for recurred lesions in 6 patients, and chemotherapeutic regimens were as follows: Treatment with temozolomide was administered to 10 patients; procarbazine, $\mathrm{CCNU}$ and vincristine (PCV) regimens were administered to 9 patients; metronomic temozolomide was administered to 3 patients; an ifosfamide, carboplatin and etoposide (ICE) regimen was administered to 1 patient. Gamma knife radiosurgery was performed on 3 patients and a single patient received additional radiation.

The patients were regularly treated using BEV (10 mg/kg body weight) alone or in combination with irinotecan $\left(125 \mathrm{mg} / \mathrm{m}^{2}\right)$ every 2 weeks. In total, 6 patients received BEV-only chemotherapy and 18 patients received $\mathrm{BEV}$ combined with irinotecan.

Treatment responses following BEV-based chemotherapy were estimated using the RANO criteria (7). The RANO criteria divides tumor responses into four types based on alterations in MRI and clinical features: Complete response (CR), partial response (PR), stable disease (SD) and progressive disease (PD). A target lesion was defined as a primary tumor site including any peritumoral edema and a new lesion was defined as a spatially-separate tumor within the brain. Radiological progression patterns were divided into dominant enhancing and non-enhancing lesions, according to the alterations in T1-enhancing and T2/FLAIR MRI images (Fig. 1). Non-enhancing lesion progression patterns were diffuse or focal. Additionally, clinical deterioration was considered a
Table I. Clinical and radiological characteristics of patients who received BEV-based chemotherapy.

A, Characteristics of all patients

\begin{tabular}{lc}
\hline Variable & $\mathrm{n}=24(\%)$ \\
\hline Gender & \\
Male & $10(41.7)$ \\
Female & $14(58.3)$ \\
Median age & 47.5 years (range, 29-69) \\
Pathology & $18(75.0)$ \\
Glioblastoma & $6(25.0)$ \\
Anaplastic astrocytoma & \\
ECOG PS & $3,9(50.0)$ \\
1,2 & $9,3(50.0)$ \\
3, 4 & \\
BEV chemotherapy & $6(25.0)$ \\
Alone & $18(75.0)$ \\
Combined with Irinotecan & \\
Treatment-associated & \\
complications (CTCAE 4.0) & \\
Neutrophil loss & \\
Grade 4 & \\
Grade 3 & \\
Grade 2 & \\
Hypertension & \\
Grade 3 & \\
Platelet loss & \\
Grade 2 & \\
Hemorrhoid bleeding & \\
Grade 2 & \\
Epistaxis & \\
Grade 1 & \\
General muscle weakness & \\
Grade 1 & \\
Grticaria & \\
\hline
\end{tabular}

$\mathrm{B}$, Response evaluation

\begin{tabular}{lc} 
Variable & $\mathrm{n}=22(\%)$ \\
\hline Best response (RANO criteria) & \\
Complete response & $3(13.6)$ \\
Partial response & $10(45.5)$ \\
Stable response & $3(13.6)$ \\
Progressive disease & $6(27.3)$ \\
Progression-free survival, & Median, 2.8 (range, 0.6-10.1) \\
months &
\end{tabular}

$\mathrm{C}$, Disease progression patterns in BEV responders

\begin{tabular}{ll}
\hline Variable & $\mathrm{n}=16(\%)$ \\
\hline Only clinical progression & $2(12.5)$ \\
Only radiological progression & $5(31.3)$ \\
Clinical and radiological progression & $9(56.2)$
\end{tabular}


Table I. Continued.

$\mathrm{D}$, Radiological disease progression in BEV responders

\begin{tabular}{lc}
\hline Variable & $\mathrm{n}=14(\%)$ \\
\hline Target lesions & \\
Dominant enhancing lesions & $8(57.1)$ \\
Dominant non-enhancing lesions & $6(42.9)$ \\
Diffuse & 3 \\
Focal & 3 \\
New lesions & 4 \\
Dominant enhancing lesions & 2 \\
Dominant non-enhancing lesions & 2 \\
\hline
\end{tabular}

E, Radiological progressive disease in BEV non-responders

\begin{tabular}{lc}
\hline Variable & $\mathrm{n}=6(\%)$ \\
\hline Target lesions & \\
Dominant enhancing lesions & $4(66.7)$ \\
Dominant non-enhancing lesions & $2(33.3)$ \\
Diffuse & 2 \\
Focal & 0 \\
New lesions & 2 \\
Predominant enhancing lesion & 1 \\
Predominant non-enhancing lesion & 1 \\
Subependymal enhancement & $9 / 20(45.0)$ \\
\hline CTCAE, National Cancer Institute Common Terminology Criteria \\
for Adverse Events version 4.0; RANO, Response Assessment in \\
Neuro-Oncology; BEV, bevacizumab. \\
\hline
\end{tabular}

recurrence pattern. Ventricular subependymal enhancement was analyzed as evidence of cerebrospinal fluid dissemination on brain imaging studies.

Toxic effects were assessed using the National Cancer Institute Common Terminology Criteria for Adverse Events version 4.0 (CTCAE), with a score of 1 indicating mild adverse effects, 2 indicating moderate adverse effects, 3 indicating severe adverse effects, 4 indicating life-threatening adverse effects and 5 denoting mortality-associated with the adverse effects. Physical examinations, including neurological exams, blood pressure measurements and laboratory tests of blood and urine, were performed biweekly. Eastern Cooperative Oncology Group (ECOG) performance status (PS) scales were used to assess patient disease progression and to determine the appropriate treatment prognosis (9).

Statistical analysis. Follow-up durations were expressed as the median average and range. The effects of single variables on progression-free survival (PFS) and overall survival (OS) rates were determined using univariate analyses. Single variables included pathologic grade, chemotherapeutic regimen, radiological progression pattern, associated subependymal enhancement and salvage treatment following BEV-based chemotherapy. PFS was calculated from the initiation of BEV-based chemotherapy to clinicoradiological disease progression; OS was calculated from the time of failure to respond to BEV-based chemotherapy to the date of mortality (or the last follow-up visit). PFS and OS probabilities were estimated using the Kaplan-Meier method and compared using log-rank tests. All statistical analyses were performed using SPSS 18.0 (SPSS, Inc., Chicago, IL, USA). P<0.05 was considered to indicate a statistically significant difference.

\section{Results}

Tumor response and toxicity of BEV-based chemotherapy. Prior to BEV-based treatment, the ECOG PS scores were 1 in 3 patients, 2 in 9 patients, 3 in 9 patients and 4 in 3 patients. In total, 2 patients discontinued treatment due to chemotherapy-associated complications that included rectal bleeding and severe hematologic toxicity following a single administration of BEV combined with irinotecan. A total of 22 patients received a median of 6 cycles (range, 2-18) of $\mathrm{BEV}$, and treatment responses were analyzed using the RANO criteria. The objective tumor response rates were $13.6 \% \mathrm{CR}, 45.5 \% \mathrm{PR}$, $13.6 \% \mathrm{SD}$ and $27.3 \% \mathrm{PD}$. It was considered that CR, PR and $\mathrm{SD}$ were BEV-responsive, but PD was BEV-non-responsive.

According to CTCAE version 4.0, patients exhibited chemotherapy-associated complications, including grade 4 $(n=2)$, grade $3(n=1)$ and grade $2(n=2)$ decreases in neutrophil counts, grade 3 hypertension $(n=1)$, grade 2 platelet loss $(n=1)$, grade 2 hemorrhoid bleeding $(n=1)$, grade 1 epistaxis $(n=2)$, grade 1 general muscle weakness $(n=3)$ and grade 1 urticaria $(n=1$; Table I). Severe grade 3 and 4 toxicities occurred in $4 / 24$ patients $(16.7 \%)$.

The median PFS was 2.8 months (range, 0.6-10.1) following BEV-based chemotherapy. Depending on the pathology, the median PFS times were 3.2 \pm 0.4 months for patients with glioblastoma and $2.7 \pm 1.5$ months for patients with anaplastic astrocytoma $(\mathrm{P}=0.482$; Fig. $2 \mathrm{~A})$. For chemotherapeutic regimens, the median PFS of patients who received BEV alone was $2.3 \pm 1.7$ months, and that for patients who received $\mathrm{BEV}$ combined with irinotecan was $2.8 \pm 0.5$ months $(\mathrm{P}=0.823$; Fig. 2B).

Patterns of disease progression following BEV-based chemotherapy. Patterns of disease progression are summarized in Table I and Fig. 1. In BEV responders, clinical progression without definitive radiological progression was observed in 2/16 patients $(12.5 \%)$. In total, 5 patients $(31.3 \%)$ exhibited only radiological progression and 9 patients (56.2\%) exhibited clinical and radiological progression.

For radiological progression in BEV responders, target lesions were dominant enhancing tumors in $8 / 14$ patients $(57.1 \%)$, and were non-enhancing tumors in $6 / 14$ patients $(42.9 \%)$. Out of the 6 patients with non-enhancing tumors, 3 had diffuse lesions and 3 had focal lesions. New lesions developed in 4 patients, of which 2 were enhancing lesions and 2 were non-enhancing lesions. For radiological disease progression in BEV non-responders, target lesions were dominant enhancing tumors in $4 / 6$ patients $(66.7 \%)$ and were diffuse non-enhancing tumors in 2 patients $(33.3 \%)$. New lesions were observed in 2 patients, of which 1 was enhancing and 1 was 


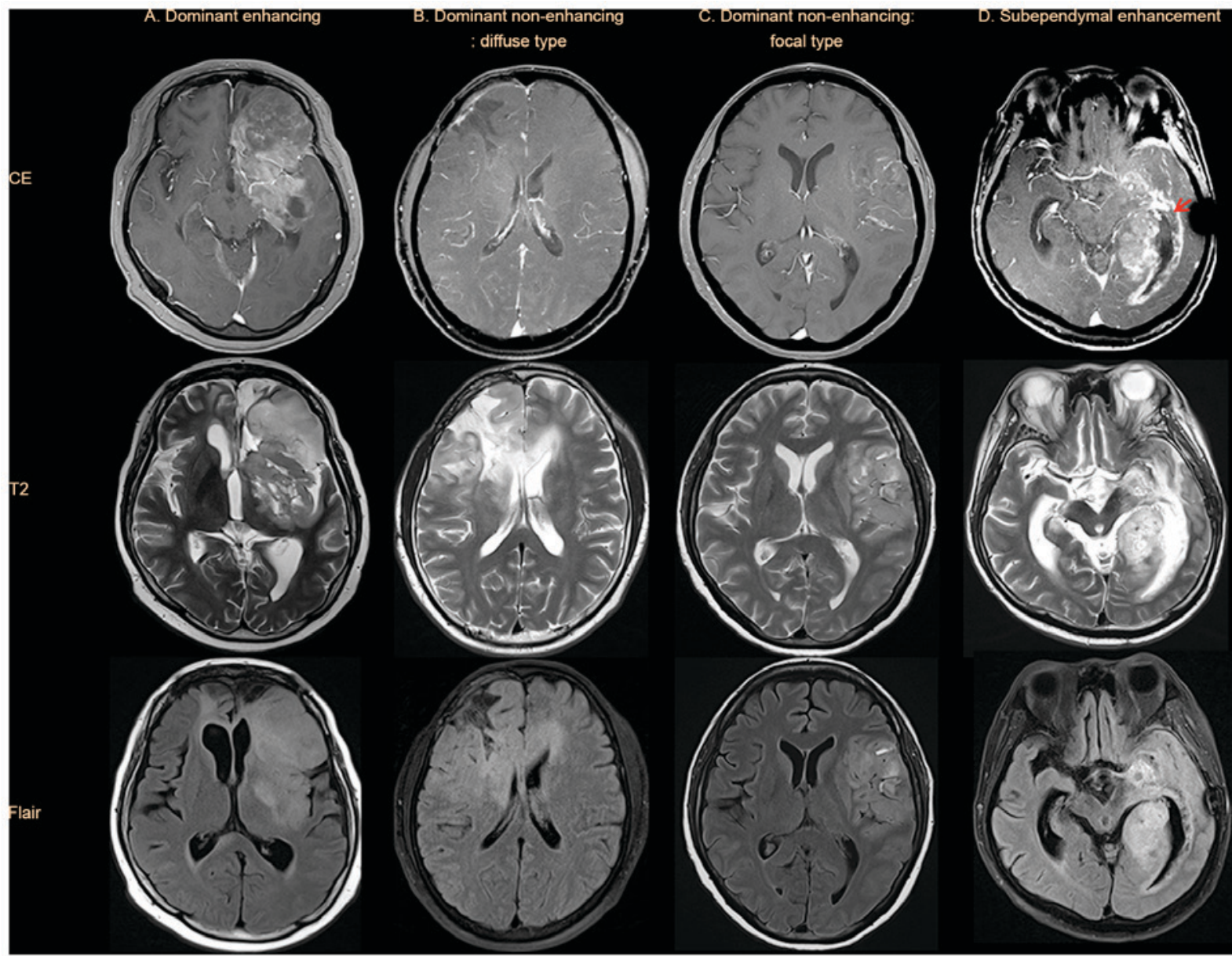

Figure 1. Radiological progression patterns according to T1-enhancing and T2/FLAIR MRI images. (A) Dominant enhancing lesion. (B) Diffuse T2/FLAIR non-enhancing lesion without an enhancement flare-up. (C) Focal T2/FLAIR non-enhancing lesion without an enhancement flare-up. (D) Associated subependymal enhancement (red arrow). CE, contrast enhancement; FLAIR, fluid attenuation inversion recovery; MRI, magnetic resonance imaging.

A

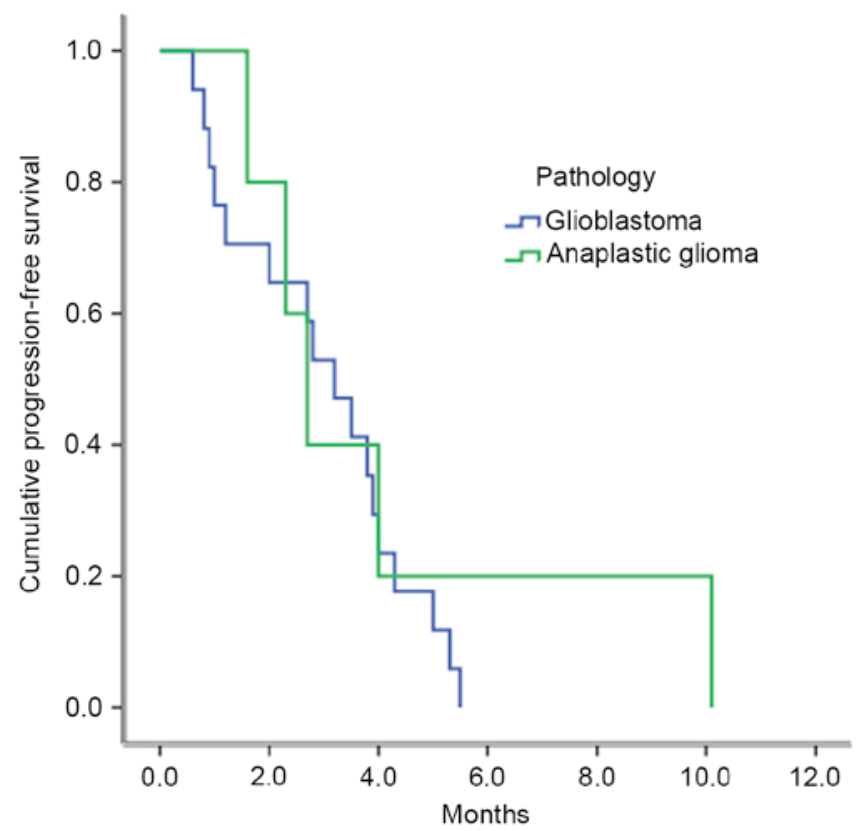

B

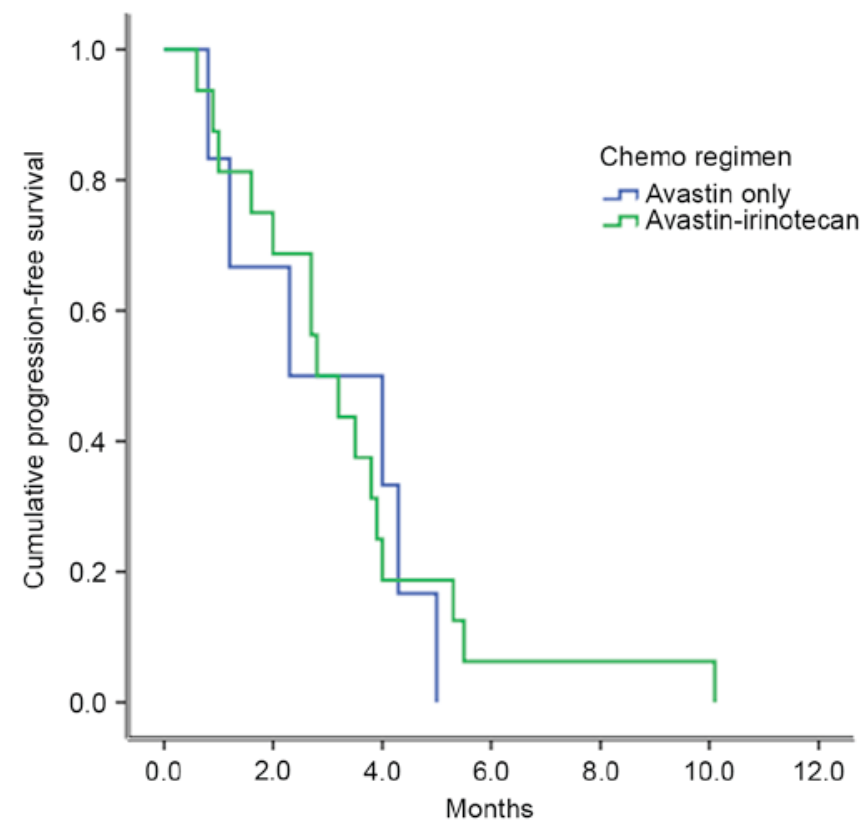

Figure 2. Kaplan-Meier curves for PFS rate. (A) Depending on pathology, the median PFS of patients with glioblastoma was $3.2 \pm 0.4$ months, and that of patients with anaplastic astrocytoma was $2.7 \pm 1.5$ months $(\mathrm{P}=0.482)$. (B) Depending on the chemotherapeutic regimen, the median PFS of patients who received $\mathrm{BEV}$ alone was $2.3 \pm 1.7$ months, and that of patients who received BEV combined with irinotecan was $2.8 \pm 0.5$ months $(\mathrm{P}=0.823)$. 


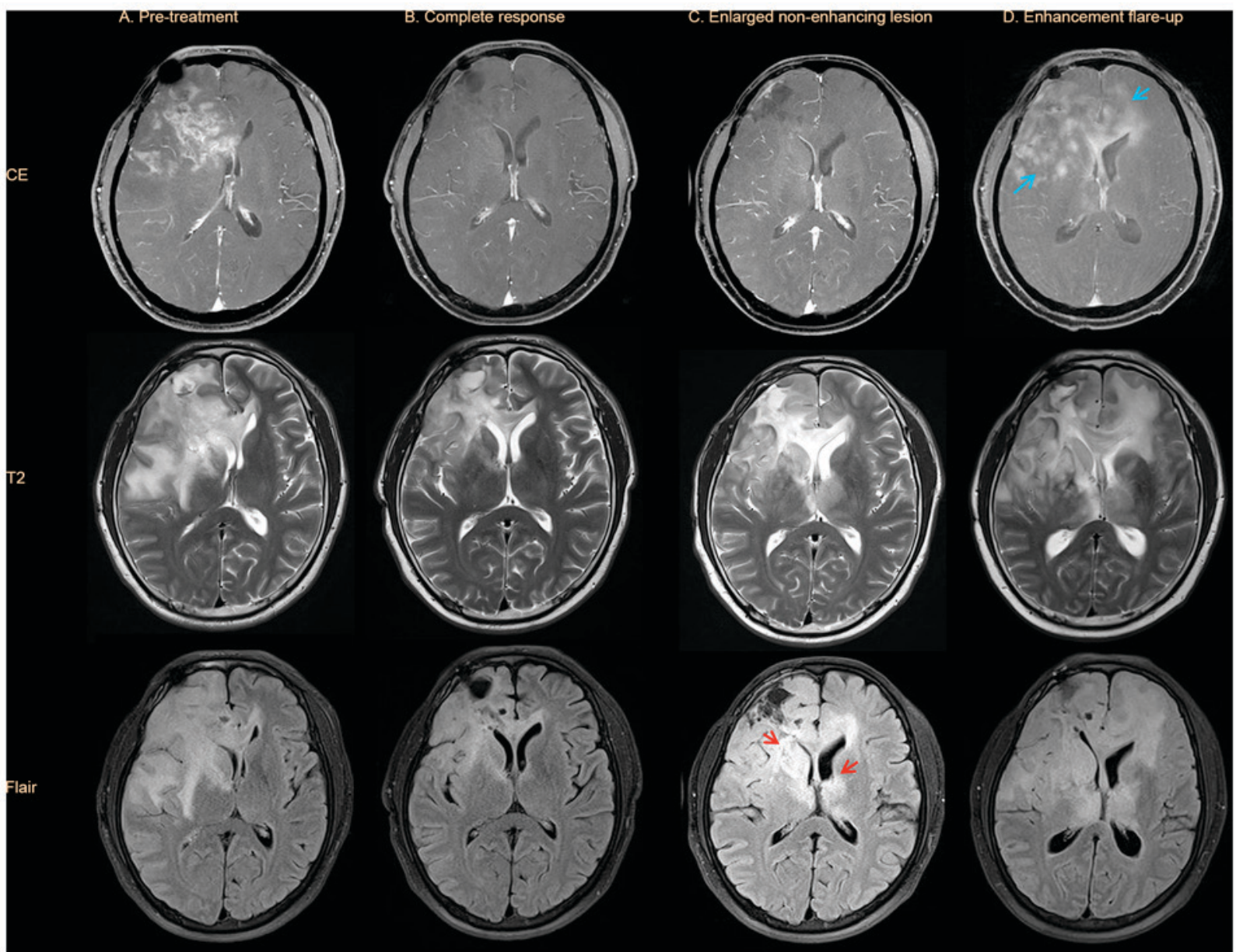

Figure 3. The radiological progression patterns in BEV complete responders. (A) The pre-treatment state is presented. (B) In a complete response following BEV-based chemotherapy, all enhancing lesions disappeared and the non-enhancing lesions improved without new lesions. (C) In radiological progression, the T2/FLAIR lesion was enlarged (red arrow) despite stable enhancing lesions. (D) In radiological progression, enlarged T2/FLAIR lesions with enhancement flare-ups (sky blue arrow) were observed. CE, contrast enhancement; BEV, bevacizumab; FLAIR, fluid attenuation inversion recovery.

non-enhancing. Subependymal enhancement was associated with $9 / 20$ patients $(45.0 \%)$, and included BEV responders and non-responders.

The radiological progression patterns in BEV complete responders. All enhancing lesions disappeared in the BEV complete responders $(n=3)$, and non-enhancing lesions improved without any new lesions developing (Fig. 3). T2/FLAIR lesions enlarged in cases of radiological progression despite stable enhancing lesions, which were associated with clinical deterioration in 2 patients and not associated with clinical deterioration in 1 patient. All enlarged T2/FLAIR lesions with enhancement flare-ups were associated with clinical deterioration.

The overall survival rate following failure of BEV-based chemotherapy. The median OS was 4.5 months (range, 0.4-34.0) following BEV-based chemotherapy failure. In total, 8 patients $(36.4 \%)$ received salvage therapies, including metronomic temozolomide $(n=3)$, ICE chemotherapy $(n=2)$, additional radiation therapy $(n=2), \operatorname{PCV}(n=1)$ and gamma knife radiosurgery $(n=1)$. A total of 14 patients received supportive care. The median OS for patients who received salvage therapy was $5.1 \pm 0.7$ months, as compared with
3.0 \pm 0.7 months for patients who received supportive care $(\mathrm{P}=0.297)$. According to radiological progression patterns, the median OS was $4.5 \pm 0.4$ months for patients with dominant enhancing lesions, $4.4 \pm 2.6$ months for patients with diffuse non-enhancing lesions and $3.3 \pm 0.8$ months for patients with focal non-enhancing lesions $(\mathrm{P}=0.749$; Fig. 4A). The median OS in patients with associated subependymal enhancement

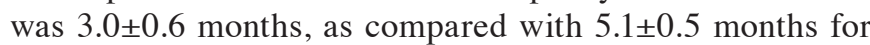
those without subependymal enhancement ( $\mathrm{P}=0.072$; Fig. 4B).

\section{Discussion}

Malignant glial tumors are highly vascular and have increased expression levels of VEGF, which binds to VEGF receptors and promotes endothelial cell proliferation and tumor angiogenesis (10). Anti-angiogenic therapy exhibits anti-tumor effects via inducing endothelial cell apoptosis, inhibiting novel vessel formation and decreasing tumor perfusion, which then results in tumor starvation (11). During initial treatment, abnormal tumor vasculature may be transiently normalized by reducing blood vessel diameter and permeability, which subsequently increases tumor perfusion, reduces interstitial pressure and improves tumor oxygenation (12). At this stage, the effects of radiotherapy 

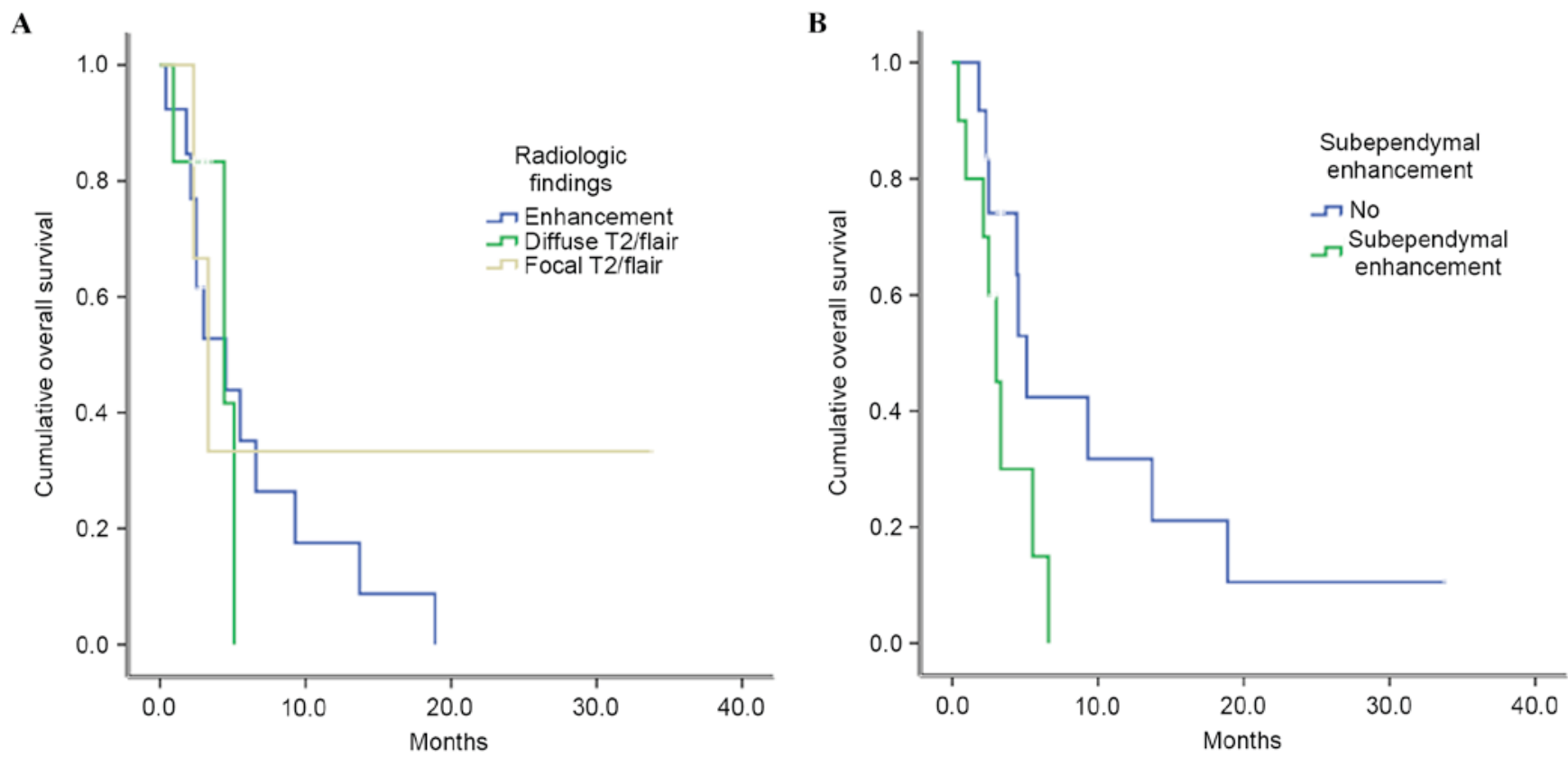

Figure 4. Kaplan-Meier curves for OS rate. (A) According to radiological progression patterns, the median OS was $4.5 \pm 0.4$ months for patients with predominant enhancing lesions, $4.4 \pm 2.6$ months for patients with diffuse non-enhancing lesions and $3.3 \pm 0.8$ months for patients with focal non-enhancing lesions $(\mathrm{P}=0.749)$. (B) The median OS for patients with associated subependymal enhancement was $3.0 \pm 0.6$ months, and $5.1 \pm 0.5$ months for patients without subependymal enhancement $(\mathrm{P}=0.072)$. PFS, progression-free survival; OS, overall survival; $\mathrm{BEV}$, bevacizumab.

and cytotoxic chemotherapeutic agents may potentially be improved (12). Augmentation of host immunity may also occur upon reducing VEGF-mediated immune suppression, which in turn improves the effects of immunotherapy (13). Augmentation of BEV-mediated benefits has been attempted using chemotherapeutic agents, targeted therapy and irradiation $(4,12,13)$. A previous study identified that BEV combined with CCNU improved the 6-month PFS rate to $41 \%$, as compared with $18 \%$ for BEV alone (4). However, these combination therapies failed to improve survival beyond that conferred by monotherapy, which is explained by the effect of BEV decreasing drug delivery to the tumor (14). Finally, adaptive resistance promotes mesenchymal transition and increased invasiveness in response to BEV treatment (6). In the present study, BEV combined with irinotecan improved survival rates, as compared with BEV alone, although this was not to a statistically significant extent.

The majority of retrospective studies suggest that BEV may modestly improve patient outcomes $(4,14,15)$. In the current study, the median PFS was 2.8 months. Responses to anti-angiogenic therapy occur rapidly, and tumor and edema volumes have been established to decrease within 6 weeks of therapy initiation $(16,17)$. Anti-angiogenic therapy targeting the VEGF signaling pathway may promptly reduce vessel permeability and contrast media extravasation. This rapid and transient radiological alteration is called a 'pseudoresponse' $(16,17)$. The decrease in edema was rapid and sustained, even following tumor progression. Tumor progression also occurs without necrosis, which differs from the recurrence patterns observed following treatment with other chemotherapeutic agents. Anti-angiogenic treatments have focused on brain tumor imaging in order to accurately define tumor responses and progression $(16,17)$.
The radiological findings of BEV-induced chemotherapy responders differ from the typical radiological results of patients who did not receive BEV-based chemotherapy, as aforementioned. Enlargement of non-enhancing lesions with decreased or stable enhancing lesions are a typical relapse progression pattern following BEV-based chemotherapy, compared with typically deteriorating enhancing lesions following non-BEV chemotherapy $(18,19)$. Flare-up of enhancing lesions and clinical deterioration without significant radiological alterations are observed in BEV chemotherapy responders. These characteristics were introduced into the revised RANO criteria (7). Enlarged T2/FLAIR lesions, despite stable enhancing lesions, are frequently present in BEV chemotherapy responders. The use of RANO criteria to assess responses would effectively estimate tumor progression, compared with other criteria that do not consider T2/FLAIR MRI results. The RANO criteria are reported to allow disease progression detection $\sim 1$ month earlier, as compared with criteria that do not account for T2/FLAIR imaging (20). However, these criteria do not state the degree of T2/FLAIR alterations required to define tumor progression, and these results may be treatment-associated alterations, including perioperative ischemia, inflammation, post-radiation demyelination and leukoencephalopathy. Furthermore, patterns of progression may differ amongst patients with malignant glioma (21). In the current study, clinical deterioration without definitive radiological alterations were detected in $12.5 \%$ of patients. Enlarged dominant enhancing lesions were noted in $57.1 \%$ of BEV-responders and non-enhancing lesions were observed in $42.9 \%$ of BEV responders. Among the radiological results, patients with tumors that had associated subependymal enhancement suggesting dissemination of tumor cells into the cerebrospinal fluid had a poor OS, as compared with patients with tumors 
lacking enhancement $(\mathrm{P}=0.072)$. Notably, the radiological progression of $\mathrm{BEV}$ complete responders primarily demonstrated progressive enlarged T2/FLAIR lesions, despite stable enhancing lesions with or without clinical deterioration. Enlarged T2/FLAIR lesions with enhancement flare-ups were associated with clinical deterioration in all patients. The progression of non-enhancing lesions may delay detection of treatment failure, particularly in BEV complete responders. This delay may potentially lead to an aggressive phenotype and be a negative prognostic factor.

Pharmacological inhibition of VEGF induces tumor hypoxia and the growth of bone marrow-derived cells, which then promotes the alternative angiogenesis pathway and tumor growth (22). Considering this, tumors may be more invasive and aggressive following anti-angiogenic treatment and, therefore, alternative salvage treatments must be considered following BEV-based chemotherapy failure. In one previous study, the median OS of patients was 5.6 months with heterogeneous salvage treatments following BEV failure, which was not reduced compared with that of the historical salvage treatments (18). In the present study, the median OS of patients was 4.5 months following BEV-based chemotherapy failure. A total of 8 patients $(36.4 \%)$ received salvage therapies, which resulted in a median 5.3 month OS compared with a 2.6 month OS in the supportive care group.

In conclusion, for patients treated with BEV-based chemotherapy, caution is required to determine tumor progression based on clinical and radiological alterations. In certain cases, clinical deterioration without radiological alterations suggested tumor progression. Additionally, tumor progression through increased non-enhancing lesions was observed in numerous BEV-responders. Clinical symptoms and radiological alterations of non-enhancing lesions must be evaluated in order to assess tumor progression in the BEV responders, particularly those patients who experienced a complete response.

\section{Acknowledgements}

This study was supported by a grant (grant no. HCRI14029-1) from the Chonnam National University Hwasun Hospital Research Institute of Clinical Medicine (Gwangju, Republic of Korea).

\section{References}

1. Levin VA, Mendelssohn ND, Chan J, Stovall MC, Peak SJ, Yee JL, Hui RL and Chen DM: Impact of bevacizumab administered dose on overall survival of patients with progressive glioblastoma. J Neurooncol 122: 145-150, 2015.

2. Friedman HS, Prados MD, Wen PY, Mikkelsen T, Schiff D, Abrey LE, Yung WK, Paleologos N, Nicholas MK, Jensen R, et al: Bevacizumab alone and in combination with irinotecan in recurrent glioblastoma. J Clin Oncol 27: 4733-4740, 2009.

3. Kreisl TN, Kim L, Moore K, Duic P, Royce C, Stroud I, Garren N, Mackey M, Butman JA, Camphausen K, et al: Phase II trial of single-agent bevacizumab followed by bevacizumab plus irinotecan at tumor progression in recurrent glioblastoma. J Clin Oncol 27: 740-745, 2009

4. Taal W, Oosterkamp HM, Walenkamp AM, Dubbink HJ, Beerepoot LV, Hanse MC, Buter J, Honkoop AH, Boerman D, de Vos FY, et al: Single-agent bevacizumab or lomustine versus a combination of bevacizumab plus lomustine in patients with recurrent glioblastoma (BELOB trial): A randomised controlled phase 2 trial. Lancet Oncol 15: 943-953, 2014.
5. Lu KV and Bergers G: Mechanisms of evasive resistance to anti-VEGF therapy in glioblastoma. CNS Oncol 2: 49-65, 2013.

6. Piao Y, Liang J, Holmes L, Henry V, Sulman E and de Groot JF: Acquired resistance to anti-VEGF therapy in glioblastoma is associated with a mesenchymal transition. Clin Cancer Res 19: 4392-4403, 2013.

7. Wen PY, Macdonald DR, Reardon DA, Cloughesy TF, Sorensen AG, Galanis E, Degroot J, Wick W, Gilbert MR, Lassman AB, et al: Updated response assessment criteria for high-grade gliomas: Response assessment in neuro-oncology working group. J Clin Oncol 28: 1963-1972, 2010.

8. Louis DN, Ohgaki H, Wiestler OD, Cavenee WK, Burger PC, Jouvet A, Scheithauer BW and Kleihues P: The 2007 WHO classification of tumours of the central nervous system. Acta Neuropathol 114: 97-109, 2007.

9. Oken MM, Creech RH, Tormey DC, Horton J, Davis TE, McFadden ET and Carbone PP: Toxicity and response criteria of the eastern cooperative oncology group. Am J Clin Oncol 5: 649-655, 1982.

10. Lamszus K, Ulbricht U, Matschke J, Brockmann MA, Fillbrandt R and Westphal M: Levels of soluble vascular endothelial growth factor (VEGF) receptor 1 in astrocytic tumors and its relation to malignancy, vascularity, and VEGF-A. Clin Cancer Res 9: 1399-1405, 2003.

11. Folkman J: Tumor angiogenesis: Therapeutic implications. N Engl J Med 285: 1182-1186, 1971.

12. Winkler F, Kozin SV, Tong RT, Chae SS, Booth MF, Garkavtsev I, Xu L, Hicklin DJ, Fukumura D, di Tomaso E, et al: Kinetics of vascular normalization by VEGFR2 blockade governs brain tumor response to radiation: Role of oxygenation, angiopoietin-1, and matrix metalloproteinases. Cancer Cell 6: 553-563, 2004.

13. Shrimali RK, Yu Z, Theoret MR, Chinnasamy D, Restifo NP and Rosenberg SA: Antiangiogenic agents can increase lymphocyte infiltration into tumor and enhance the effectiveness of adoptive immunotherapy of cancer. Cancer Res 70: 6171-6180, 2010.

14. Van der Veldt AA, Lubberink M, Bahce I, Walraven M, de Boer MP, Greuter HN, Hendrikse NH, Eriksson J, Windhorst AD, Postmus PE, et al: Rapid decrease in delivery of chemotherapy to tumors after anti-VEGF therapy: Implications for scheduling of anti-angiogenic drugs. Cancer Cell 21: 82-91, 2012.

15. Batchelor TT, Reardon DA, de Groot JF, Wick W and Weller M: Antiangiogenic therapy for glioblastoma: Current status and future prospects. Clin Cancer Res 20: 5612-5619, 2014.

16. Pope WB, Lai A, Nghiemphu P, Mischel P and Cloughesy TF: MRI in patients with high-grade gliomas treated with bevacizumab and chemotherapy. Neurology 66: 1258-1260, 2006.

17. Ananthnarayan S, Bahng J, Roring J, Nghiemphu P, Lai A, Cloughesy $\mathrm{T}$ and Pope WB: Time course of imaging changes of GBM during extended bevacizumab treatment. J Neurooncol 88: 339-347, 2008.

18. Iwamoto FM, Abrey LE, Beal K, Gutin PH, Rosenblum MK, Reuter VE, DeAngelis LM and Lassman AB: Patterns of relapse and prognosis after bevacizumab failure in recurrent glioblastoma. Neurology 73: 1200-1206, 2009.

19. Gaspar LE, Fisher BJ, Macdonald DR, LeBer DV, Halperin EC, Schold SC Jr and Cairncross JG: Supratentorial malignant glioma: Patterns of recurrence and implications for external beam local treatment. Int J Radiat Oncol Biol Phys 24: 55-57, 1992.

20. Gallego Pérez-Larraya J, Lahutte M, Petrirena G, Reyes-Botero G, González-Aguilar A, Houillier C, Guillevin R, Sanson M, Hoang-Xuan K and Delattre JY: Response assessment in recurrent glioblastoma treated with irinotecan-bevacizumab: Comparative analysis of the Macdonald, RECIST, RANO, and RECIST + F criteria. Neuro Oncol 14: 667-673, 2012.

21. Nowosielski M, Wiestler B, Goebel G, Hutterer M, Schlemmer HP, Stockhammer G, Wick W, Bendszus M and Radbruch A: Progression types after antiangiogenic therapy are related to outcome in recurrent glioblastoma. Neurology 82: 1684-1692, 2014.

22. Du R, Lu KV, Petritsch C, Liu P, Ganss R, Passegué E, Song H, Vandenberg S, Johnson RS, Werb Z and Bergers G: HIFlalpha induces the recruitment of bone marrow-derived vascular modulatory cells to regulate tumor angiogenesis and invasion. Cancer Cell 13: 206-220, 2008. 